\title{
BMJ Open Association between bipolar spectrum disorder and bone health: a meta- analysis and systematic review protocol
}

\author{
Vinoomika Chandrasekaran, ${ }^{1}$ Sharon L Brennan-Olsen, ${ }^{1,2,3,4}$ Amanda L Stuart, \\ Julie A Pasco, ${ }^{1,2}$ Michael Berk, ${ }^{1,5,6,7,8}$ Jason M Hodge, ${ }^{1,8}$ Lana J Williams ${ }^{1}$
}

To cite: Chandrasekaran V, Brennan-Olsen SL, Stuart AL, et al. Association between bipolar spectrum disorder and bone health: a metaanalysis and systematic review protocol. BMJ Open 2017:7:e013981.

doi:10.1136/bmjopen-2016013981

- Prepublication history for this paper is available online. To view these files please visit the journal online (http://dx.doi.org/10.1136/ bmjopen-2016-013981).

Received 23 August 2016 Revised 29 November 2016 Accepted 20 December 2016

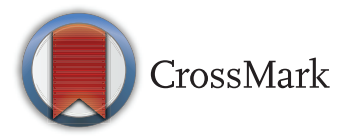

For numbered affiliations see end of article.

Correspondence to Associate Professor Lana J Williams; lanaw@barwonhealth.org.au

\section{ABSTRACT}

Introduction: Bipolar spectrum disorder is a chronic, episodic illness, associated with significant personal, social and economic burden. It is estimated to affect $\sim 2.4 \%$ of the population worldwide and is commonly associated with psychological and/or physiological comorbidities. Osteoporosis is one such comorbidity, a disease of bone that is asymptomatic until a fracture occurs. This systematic review attempts to capture, collate, assess and discuss the literature investigating the association between bipolar spectrum disorder and bone health.

Methods and analysis: We aim to identify articles that investigate the association between bipolar spectrum disorder and bone health in adults by systematically searching the MEDLINE, PubMed, OVID and CINAHL databases. Two independent reviewers will determine eligibility of studies according to predetermined criteria, and methodological quality will be assessed using a previously published scoring system. A meta-analysis will be conducted, and statistical methods will be used to identify and control for heterogeneity, if possible. If numerical syntheses are prevented due to statistical heterogeneity, a best evidence synthesis will be conducted to assess the level of evidence for associations between bipolar spectrum disorder and bone health.

Ethics and dissemination: Ethical permission will not be required for this systematic review since only published data will be used. This protocol will be registered with PROSPERO. Findings of the review will be published in a peer-reviewed scientific journal, and will be presented to clinical and population health audiences at national and international conferences.

\section{INTRODUCTION}

Bipolar spectrum disorder, a mental disorder characterised by biphasic fluctuations in mood, is a severe, chronic, episodic illness, which generally necessitates pharmacotherapy and/or psychotherapy. It is estimated to affect $\sim 2.4 \%$ of the population ${ }^{1}$ and has been ranked the sixth leading cause of disability in the world, among individuals aged

\section{Strengths and limitations of this study}

This systematic review will explore a novel and covert clinical area.

- It will comprehensively assess existing literature that investigates associations between bipolar spectrum disorder and bone health.

- Potential confounders and/or mediators of the relationship will be identified.

- Two authors will independently confirm study selection, and undertake data extraction and methodological assessment.

- A potential limitation of this review may be the paucity of data available due to this being a nascent area of enquiry, and that there may be much heterogeneity in available studies.

15-44 years. ${ }^{2}$ The related direct and indirect costs associated with bipolar spectrum disorder are substantial. ${ }^{3}{ }^{4}$ The burden of bipolar spectrum disorder is experienced on many levels-by the sufferer, their immediate family and friends and also by the healthcare system. Symptom burden and disease course is often worsened in the presence of psychological and/or physiological comorbidities. ${ }^{5} 6$

Psychiatric disorders, including bipolar spectrum disorder, have been associated with early mortality, with $\sim 60 \%$ of this excess mortality due to chronic physical illness. ${ }^{7}$ A particularly common comorbidity of unipolar depression is osteoporosis. ${ }^{8}{ }^{9}$ Yet it is normatively overlooked, due to being asymptomatic until fracture occurs. Osteoporosis is a global public health issue, estimated to affect nearly 49 million individuals in industrialised countries, with this on the rise as a consequence of the ageing population. ${ }^{10} 11$ The rising global economic burden related to the direct and indirect costs of medical care and rehabilitation of individuals with osteoporotic fractures is concerning. ${ }^{12}{ }^{13}$ Both clinically diagnosed unipolar depression and depressive symptoms have been shown to be 
associated with deficits in bone mineral density (BMD), bone loss over time and increased fracture risk in men and women. ${ }^{91415}$ Furthermore, antidepressants, in particular, selective serotonin reuptake inhibitors used in treatment of depression, have also been shown to be noxious to bone. ${ }^{16}$ Other psychotropic medication, namely antipsychotics and anticonvulsants, have also been shown to have a deleterious effect on bone. ${ }^{17-19} \mathrm{~A}$ recent research synthesis with meta-analyses concluded that depression should be considered a serious risk factor for osteoporosis, based on aggregated data showing BMD among individuals with depression to be up to $7.3 \%$ lower. ${ }^{15}$ Another meta-analysis reported depression to be associated with up to a $52 \%$ increased risk of fracture. ${ }^{21}$ Whether this is true for bipolar spectrum disorder per se is yet to be determined.

Considering the previous research discussing the probable association between unipolar depression and bone, this review would essentially provide a starting point for similar investigations in bipolar spectrum disorder. This review will analyse the existing data, and this information may provide a clearer background into bone fragility associated with bipolar spectrum disorder, enabling the details of this association to be further explored.

\section{Objectives}

This systematic review will:

1. Identify published studies that investigate the association between bipolar spectrum disorder and bone health, including BMD and fracture;

2. Evaluate the quality of the methodology used in each of the studies eligible for inclusion in this review;

3. Collate the evidence, including identifying any potential confounding and/or mediating factors in the association between bipolar spectrum disorder and bone health;

4. Perform sensitivity analyses to account for differences between (a) self-reported and diagnosed bipolar spectrum disorder, (b) diagnostic criteria between versions of the Diagnostic and Statistical Manual (DSM) and/or International Classification of Diseases (ICD) and (c) bipolar disorders I and II;

5. Provide a comprehensive synthesis of the findings using previously published methodology.

\section{METHODS}

\section{Criteria for considering studies for this review}

Articles resulting from cross-sectional, case-control and/ or longitudinal studies of bone health (defined as BMD, bone quality, osteoporosis and/or fracture), in adult populations ( $\geq 18$ years) with bipolar spectrum disorder (defined by self-report, medical records or diagnoses based on any version of the Diagnostic and Statistical Manual of Mental Disorders or International Statistical Classification of Diseases and Related Health Problems criteria), inclusive of any sex or nationality, and published in any year, will be considered as eligible for this review.

Grey literature, case studies, theses and conference presentations will be excluded. Baseline data from randomised control trials will be included and treated as cross-sectional analyses.

\section{Search strategy and data extraction}

In order to identify the relevant literature, we will undertake an electronic search strategy to investigate research databases from the disciplines of medical, health and the social sciences (PubMed, OVID, CINAHL, MEDLINE). The following medical subject headings will be applied: "bipolar disorder" AND ("bone" OR "osteoporosis" OR "fracture" OR "bone density"), to identify publications that match our eligibility criteria. For our search strategy, we will also include the key word term of 'bipolar spectrum disorder'.

No limits will be applied with regard to year of publication. For each database, where appropriate, relevant truncation will be applied. One reviewer will apply the search strategy and identify eligible literature for inclusion by cross-checking with the predetermined eligibility criteria. Two further reviewers will confirm the eligibility of those identified articles. Professional assistance would be sought to interpret articles written in languages other than English, in order to confirm their relevance to the eligibility criteria. Finally, the reference lists of eligible studies will be manually searched by two reviewers. ${ }^{22}$

\section{Assessment of methodological quality of included articles} The methodological scoring system of Lievense et al ${ }^{23}$ will be employed to assess the methodological quality of included articles (tables 1 and 2). Based on those methodological assessment criteria, each eligible study will be scored, with each study given either a positive or negative score for each criterion. This process of scoring methodological quality reflects cohort studies as the most optimal study design, followed by case-control studies and, finally, cross-sectional study designs. Two reviewers will independently score the methodological quality of each study; should these scores differ, the reviewers will attempt to reconcile any differences, after which a third reviewer would provide final judgement, if necessary. Each study will be ranked according to their total score (\%), and deemed as having higher methodological quality if scored above the median, as previously published. ${ }^{24}$

For the meta-analyses, we will determine the population with bipolar spectrum disorder to be our proxy 'treatment' group and apply Hunter-Schmidt's approach,$^{25}$ whereby a pooled within-group SD will be used. Effect size will be corrected for measurement error by dividing the effect size by the square root of the reliability coefficient of the dependent variable, whereby measurement error correction equals the effect size divided by the square root of $r$. 
Table 1 Criteria list for assessment of study quality, adapted from Lievense et a ${ }^{3}$

\begin{tabular}{|c|c|c|}
\hline Item & Criterion & $\mathrm{C} / \mathrm{CC} / \mathrm{CS}$ \\
\hline \multicolumn{3}{|c|}{ Study population } \\
\hline 1 & $\begin{array}{l}\text { Uniform point (selection before disease } \\
\text { was present) }\end{array}$ & $\mathrm{C} / \mathrm{CC} / \mathrm{CS}$ \\
\hline 2 & $\begin{array}{l}\text { Cases and controls drawn from the } \\
\text { same population }\end{array}$ & $\mathrm{CC}$ \\
\hline 3 & $\begin{array}{l}\text { Participation rate }>80 \% \text { for cases/ } \\
\text { cohort }\end{array}$ & $\mathrm{C} / \mathrm{CC} / \mathrm{CS}$ \\
\hline 4 & Participation rate $>80 \%$ for controls & $\mathrm{CC}$ \\
\hline \multicolumn{3}{|c|}{ Assessment of risk factor } \\
\hline 5 & Exposure assessment blinded & $\mathrm{C} / \mathrm{CC} / \mathrm{CS}$ \\
\hline 6 & $\begin{array}{l}\text { Exposure measured identically for } \\
\text { cases and controls }\end{array}$ & $\mathrm{CC}$ \\
\hline 7 & $\begin{array}{l}\text { Exposure assessed prior to the } \\
\text { outcome }\end{array}$ & $\mathrm{C} / \mathrm{CC} / \mathrm{CS}$ \\
\hline \multicolumn{3}{|c|}{ Assessment of outcome } \\
\hline 8 & $\begin{array}{l}\text { Bone health assessed identically in } \\
\text { patients with bipolar spectrum disorder. }\end{array}$ & $\mathrm{C} / \mathrm{CC} / \mathrm{CS}$ \\
\hline 9 & $\begin{array}{l}\text { Presence of osteoporosis assessed } \\
\text { reproducibly }\end{array}$ & $\mathrm{C} / \mathrm{CC} / \mathrm{CS}$ \\
\hline 10 & $\begin{array}{l}\text { Osteoporosis identification assessed } \\
\text { according to BMD measurements }\end{array}$ & $\mathrm{C} / \mathrm{CC} / \mathrm{CS}$ \\
\hline \multicolumn{3}{|c|}{ Study design } \\
\hline 11 & Prospective design used & $\mathrm{C} / \mathrm{CC}$ \\
\hline 12 & Follow-up time $>24$ months & C \\
\hline & Withdrawals <20\% & C \\
\hline \multicolumn{3}{|c|}{ Analysis and data presentation } \\
\hline 14 & Appropriate analysis techniques used & $\mathrm{C} / \mathrm{CC} / \mathrm{CS}$ \\
\hline 15 & Adjusted for at least age and sex & $\mathrm{C} / \mathrm{CC} / \mathrm{CS}$ \\
\hline
\end{tabular}

\section{Presenting and reporting results}

PRISMA guidelines ${ }^{26}$ will be adhered to, with regard to the presentation of findings from this review, and this protocol adheres to the PRISMA-P guidelines. ${ }^{27}$ Numbers and reasons pertaining to inclusion versus exclusion of papers in the context of the predetermined eligibility criteria will be presented in a QUOROM diagram. ${ }^{28}$ Key information regarding factors involved in the association between bipolar spectrum disorder and bone health will be identified; these factors may include, but will not be limited to, inflammatory markers, lifestyle behaviours, socioeconomic status, medications and substance use. Our findings will be useful to inform and reach a consensus as to the link between bipolar spectrum disorder and bone health.

A meta-analysis is planned; however, if a numerical synthesis is not possible due to methodological heterogeneity, a 'best evidence synthesis' will be undertaken. A 'best evidence synthesis' would evaluate the level of evidence identified, ranging from no evidence to strong evidence (table 2), as previously published in the musculoskeletal field. ${ }^{24}$

We will also perform sensitivity analyses to account for differences between (1) self-reported and diagnosed
Table 2 Method for determining the level of evidence for best evidence synthesis, adapted from Lievense et al; replicated from Brennan et $a^{P^{4}}$

\begin{tabular}{ll}
\hline Level of & Criteria for inclusion in best evidence \\
evidence & synthesis \\
Strong evidence & Generally consistent findings in: \\
& Multiple high-quality cohort studies \\
Moderate & Generally consistent findings in: \\
evidence & 1 high-quality cohort study and $>2$ \\
& high-quality case-control studies \\
& $>3$ high-quality case-control studies \\
Limited & Generally consistent findings in: \\
evidence & A single cohort study \\
& 1 or 2 case-control studies or \\
Conflicting & Inconsistent findings in $>25 \%$ of the \\
evidence & trials \\
No evidence & No studies could be found \\
\hline
\end{tabular}

bipolar spectrum disorder, (2) diagnostic criteria between versions of the DSM and/or ICD and (3) bipolar disorders I and II.

\section{Dissemination}

This protocol will be registered with PROSPERO, an international database of health-related systematic review protocols. The findings of our systematic review will be published in a peer-reviewed scientific journal, and results will be shared at national and/or international conferences relevant to the field of bipolar spectrum disorder and/or bone health.

\section{Ethics}

Since only published data will be used in this systematic review, we do not require ethical permission. However, ethical and governance standards will be strictly adhered to, in matters of data management and in the presentation and discussion of our results.

\section{Conclusion}

To the best of our knowledge, this will be the first systematic review to identify and evaluate the existing evidence base regarding associations between bipolar spectrum disorder and bone health; and determining the nature of this relationship has both public health and clinical implications. The findings of this review will contribute to existing literature investigating other psychiatric disorders and bone health, and will also provide an evidence base on which resource allocation and clinical and public health strategies aimed at reducing burden associated with both osteoporosis and bipolar spectrum disorder can be founded.

\section{Author affiliations}

${ }^{1}$ Deakin University, Geelong, Victoria, Australia

${ }^{2}$ Australian Institute for Musculoskeletal Science (AIMSS), The University of Melbourne and Western Health, St Albans, Australia 
${ }^{3}$ Department of Medicine, The University of Melbourne-Western Precinct, St Albans, Australia

${ }^{4}$ Institute for Health and Ageing, Australian Catholic University, Melbourne, Victoria, Australia

${ }^{5}$ Department of Psychiatry, University of Melbourne, Parkville, Victoria, Australia

${ }^{6}$ Florey Institute of Neuroscience and Mental Health, Parkville, Victoria, Australia

${ }^{7}$ Orygen the National Centre of Excellence in Youth Mental Health, Parkville, Victoria, Australia

${ }^{8}$ Barwon Health University Hospital, Geelong, Australia

Contributors All authors conceptualised the research question for this protocol and edited and revised the research question. VC, SLB-0 and LJW developed the e-search strategy. All authors edited, revised and approved the methodological processes. VC, SLB-0 and LJW drafted the manuscript, and all authors edited and contributed to the writing of this paper. All authors read and approved the final version, and guarantee the review.

Funding The study is supported by the National Health and Medical Research Council (NHMRC), Australia (1104438). VC is supported by a Deakin University Postgraduate Research Scholarship, SLB-0 is supported by an NHMRC Career Development Fellowship (1107510), MB is supported by an NHMRC Senior Principal Research Fellowship (1059660) and LJW is supported by an NHMRC Career Development Fellowship (1064272).

Competing interests SLB-0 has received speaker fees from Amgen, and Grant/Research support from the University of Melbourne, Deakin University, Arthritis Victoria, Arthritis Australia, Australian Association of Gerontology, and the City of Greater Geelong. JAP has received speaker fees from Amgen, Eli Lilly and Sanofi-Aventis and funding from the Geelong Region Medical Research Foundation, Barwon Health, Perpetual Trustees, The University of Melbourne, Deakin University, ANZ Charitable Trust, the American Society for Bone and Mineral Research, Amgen (Europe) GmBH, the BUPA Foundation, Osteoporosis Australia, Australia and New Zealand Bone and Mineral Society and the NHMRC. MB has received Grant/Research Support from the NIH, Simons Foundation, CRC for Mental Health, Stanley Medical Research Institute, MBF, NHMRC, Beyond Blue, Geelong Medical Research Foundation, Bristol Myers Squibb, Eli Lilly, Glaxo SmithKline, Organon, Novartis, Mayne Pharma, Servier and Astra Zeneca, and he has been a paid consultant for Astra Zeneca, Bristol Myers Squibb, Eli Lilly, Glaxo SmithKline, Janssen Cilag, Lundbeck and Pfizer and a paid speaker for Astra Zeneca, Bristol Myers Squibb, Eli Lilly, Glaxo SmithKline, Janssen Cilag, Lundbeck, Organon, Pfizer, Sanofi Synthelabo, Solvay and Wyeth. LJW has received Grant/Research support from Eli Lilly, Pfizer, The University of Melbourne, Deakin University and the NHMRC

Provenance and peer review Not commissioned; externally peer reviewed.

Open Access This is an Open Access article distributed in accordance with the Creative Commons Attribution Non Commercial (CC BY-NC 4.0) license, which permits others to distribute, remix, adapt, build upon this work noncommercially, and license their derivative works on different terms, provided the original work is properly cited and the use is non-commercial. See: http:// creativecommons.org/licenses/by-nc/4.0/

\section{REFERENCES}

1. Merikangas KR, Jin R, He JP, et al. Prevalence and correlates of bipolar spectrum disorder in the world mental health survey initiative. Arch Gen Psychiatry 2011;68:241-51.

2. Murray CL, Lopez AD. Summary: global burden of disease. In: Global burden of disease and injury series. Boston, MA, USA: The harvard school of public health, on behalf of the world health organization and the world bank, 1996:20-7.

3. Laxman KE, Lovibond KS, Hassan MK. Impact of bipolar disorder in employed populations. Am J Manag Care 2008;14:757-64.
4. Fajutrao L, Locklear J, Priaulx J, et al. A systematic review of the evidence of the burden of bipolar disorder in Europe. Clin Pract Epidemiol Ment Health 2009;5:3-3.

5. Forty L, Ulanova A, Jones L, et al. Comorbid medical illness in bipolar disorder. Br J Psychiatry 2014;205:465-72.

6. Parker GB. Comorbidities in bipolar disorder: models and management. Med J Aust 2010;193:S18-20.

7. Hert DE, Correll M, Bobes CU, et al. Physical illness in patients with severe mental disorders. I. Prevalence, impact of medications and disparities in healthcare. World Psychiatry 2011;10:52-77.

8. Rizzoli R, Cooper C, Reginster JY, et al. Antidepressant medications and osteoporosis. Bone 2012;51:606-13.

9. Aloumanis K, Mavroudis K. The "depressive" face of osteoporosis and the "osteoporotic" face of depression. Hormones (Athens) 2013;12:350-62.

10. Wade SW, Strader C, Fitzpatrick LA, et al. Estimating prevalence of osteoporosis: examples from industrialized countries. Arch Osteoporos 2014;9:1-10.

11. Estimating the prevalence of osteoporosis in Australia, AlHW, Ed. Australian institute of health and welfare. Canberra: ACT 2014:16-18.

12. Blume SW, Curtis JR. Medical costs of osteoporosis in the elderly Medicare population. Osteoporos Int 2011;22:1835-44.

13. Watts JJ, Abimanyi-Ochom J, Sanders KM. Osteoporosis costing all Australians A new burden of disease analysis-2012 to 2022. 2012. (04/08/2016). http://www.osteoporosis.org.au/sites/default/files/ files/Burden\%20of\%20Disease\%20Analysis\%202012-2022.pdf

14. Fernandes BS, Hodge JM, Pasco JA, et al. Effects of depression and serotonergic antidepressants on bone: mechanisms and implications for the treatment of depression. Drugs Ageing 2016;33:21-5.

15. Schweiger JU, Schweiger U, Hüppe M, et al. Bone density and depressive disorder: a meta-analysis. Brain and Behavior 2016;6: e00489.

16. Bruyère $O$, Reginster JY. Osteoporosis in patients taking selective serotonin reuptake inhibitors: a focus on fracture outcome. Endocrine 2015;48:65-8.

17. Yang J, Joe SH, Lee MS, et al. Effects of long-term combination treatment with valproate and atypical antipsychotics on bone mineral density and bone metabolism in premenopausal patients with bipolar disorder: a preliminary study. Psychiatry Investig 2011;8:256-61.

18. Lee $\mathrm{RH}$, Lyles $\mathrm{KW}$, Colon-Emeric $\mathrm{C}$. A review of the effect of anticonvulsant medications on bone mineral density and fracture risk. Am J Geriatr Pharmacother 2010;8:34-46.

19. Mezuk B, Morden NE, Ganoczy D, et al. Anticonvulsant use, bipolar disorder, and risk of fracture among older adults in the Veterans health administration. Am J Geriatr Psychiatry 2010;18:245-55.

20. Cizza G, Primma S, Coyle M, et al. Depression and osteoporosis: a research synthesis with meta-analysis. Horm Metab Res 2010;42:467-82.

21. Wu Q, Liu J, Gallegos-Orozco JF, et al. Depression, fracture risk and bone loss: a meta-analysis of cohort studies. Osteoporos Int 2010;21:1627-35.

22. Horsley T, Dingwall O, Sampson M. Checking reference lists to find additional studies for systematic reviews. Cochrane Database Syst Rev 2011;(8):Mr000026.

23. Lievense A, Bierma-Zeinstra S, Verhagen A, et al. Influence of work on the development of osteoarthritis of the hip: a systematic review. $J$ Rheumatol 2001;28:2520-8.

24. Brennan SL, Pasco JA, Urquhart DM, et al. The association between urban or rural locality and hip fracture in community-based adults: a systematic review. J Epidemiol Community Health 2010;64:656-65.

25. Montgomery GH, Hunter John E, Schmidt Frank L. (1990). Methods of meta-analysis: correcting error and bias in research findings. Newbury Park, CA: Sage, 592 Pages, \$38.00 (Paper). Am J Clin Hypn 2000;43:81-2.

26. Moher D, Liberati A, Tetzlaff J, et al. Preferred Reporting Items for Systematic Reviews and Meta-Analyses: the PRISMA Statement. PLoS Med 2009;6:e1000097.

27. Shamseer L, Moher D, Clarke M, et al. Preferred reporting items for systematic review and meta-analysis protocols (PRISMA-P) 2015: elaboration and explanation. BMJ 2015;349:g7647.

28. Moher D, Cook DJ, Eastwood S, et al. Improving the quality of reports of meta-analyses of randomised controlled trials: the QUOROM statement. Lancet 1999;354:1896-900. 